\title{
Spectral Intensity Variation by the Correlation Function of Refractive Index Fluctuations of the Liquid Medium
}

\author{
Nageshwar Singh \\ Magnetic and Superconducting Materials Section, Materials \& Advanced Accelerator Sciences Division, \\ Raja Ramanna Centre for Advanced Technology, Indore 452013, Madhya Pradesh, India
}

Correspondence should be addressed to Nageshwar Singh; nageshwar@rrcat.gov.in

Received 13 May 2013; Accepted 15 August 2013

Academic Editor: Ivan Djordjevic

Copyright (c) 2013 Nageshwar Singh. This is an open access article distributed under the Creative Commons Attribution License, which permits unrestricted use, distribution, and reproduction in any medium, provided the original work is properly cited.

It is proposed that a macroscopic theory of propagation and scattering of light through random media can be functional for the dye liquid flowing media in the microscopic levels too, with modest approximations. Maxwell's equation for a random refractive index medium is approximated and solved for the electric field. An analytical expression for the spectral intensity of the field scattered by the refractive index fluctuations inside a medium has been derived which was valid within the first Born approximation. Far field spectral intensity variation of the radiation propagating through the liquid medium is a consequence of variation in correlation function of the refractive index inhomogeneities. The strength of radiation scattered in a particular direction depends on the spatial correlation function of the refractive index fluctuations of the medium. An attempt is made to explain some of the experimentally observed spectral intensity variations, particularly dye emission propagation through liquid flowing medium, in the presence of thermal and flow field.

\section{Introduction}

Scattering of light is regarded as an interaction of light with matter, which leads to a change of the direction and/or the length of the incident light wave. The incident light is deflected by collisions with particles or centers of inhomogeneity of the medium. Scattering of light occurs as an effect of heterogeneity in optical properties of the medium. Wave propagation and scattering of light through a medium are a subject of investigation that has a long history. Examination of medium by wave propagation and scattering techniques is important because it provides a tool in the study of the structure of the environment and its effects on communication through it and the detection and identification of objects in various environments. In probing the medium, we may distinguish the following two types of interactions, spectroscopic and refractive index fluctuation. In the spectroscopic interaction, the molecules in the liquid react with the wave and cause absorption and scattering of the wave. The absorption and scattering cross-sections are dependent on the properties of the specific molecule, the frequency, and the environment (density and temperature). In the macroscopic interaction, the response of the medium to the incident wave is to alter the direction and spatial coherence of the photons. Large size characteristic length of medium fluctuations provides small deflection to the incident radiation, and small size gives large scattering.

The propagation of radio, acoustic, and optical waves through random media was a subject matter of investigation due to the practical interest, particularly in terrestrial, atmospheric, and ionospheric (e.g., plasma) media [1-12]. Also, it was of interest in connection with work in optical communications, imaging, and targeting systems to understand the effects of turbulence on the propagation of beams [1317], in order to minimize the beams distortion as little as possible by the presence of the fluidic media. Thus, most of the works were on the problem of wave propagation and scattering in the atmosphere, the ocean, and biological media, which are, in general, randomly varying in time and space so that the amplitude and phase of the waves may also fluctuate randomly in time and space. Jannson et al. [18] derived the expression for the spectral intensity of the field, produced by scattering of radiation of any state of coherence, in the far zone. They showed that the spectral intensity depends on 
the spatial correlation function of the field and degree of spatial coherence of the incident field. The field generated by scattering of light from a quasi-homogeneous source on a quasi-homogeneous random medium was investigated by Visser et al. [19]. The far field generated by scattering of a plane monochromatic wave incident on a quasi-homogeneous random medium was explained with the help of correlation function of the scattering potential of the random scatterer [19]. A theory was developed [20] for the fluctuations in the phase and amplitude of a laser beam probing a locally homogeneous and anisotropic medium with the help of correlation functions, which are related to the corresponding stochastic properties of the scattering medium.

Most of the theoretical and experimental works reported in the literatures are related to the coherent/incoherent or partially coherent beam propagation through macroscopic homogeneous, quasi-homogeneous, and random and/or turbulent gaseous media. However, microscopic fluctuations induced by flowing media and their effects on beam passing through them have been overlooked. To the best of our knowledge, no report is available on the spectral intensity variation by the correlation function of the refractive index fluctuation of the microscopic liquid media, particularly dye emission propagation through inhomogeneous liquid gain medium. In this paper, it is proposed that a macroscopic theory of propagation and scattering of light through random media can be functional for the dye liquid flowing media in the microscopic levels too, with a modest approximation.

Contributions of the paper can be summarized as follows: it is established that theory of propagation and scattering of light through random media can be used for the liquid flowing media within the first Born approximation. Analytical expression for the spectral intensity of the radiation scattered in terms of the correlation function of the refractive index of the inhomogeneous medium is derived, starting from Maxwell's field equation. An attempt is made to explain the experimentally observed spectral intensity variation of the fluorescence emission of dye and the dye laser emission in liquid media in the presence of thermal and flow field.

\section{Theory of Spectral Intensity Variation by Refractive Index Fluctuations of the Medium}

Let us consider wave propagation through a medium occupying a finite domain. The physical properties of the medium may be characterized by the dielectric constant $\varepsilon$. From Maxwell's equation,

$$
\operatorname{div} \varepsilon \vec{E}=\varepsilon \operatorname{div} \vec{E}+\vec{E} \cdot \operatorname{grad} \varepsilon=0 .
$$

We have

$$
\operatorname{div} \vec{E}=-\frac{\vec{E} \cdot \operatorname{grad} \varepsilon}{\varepsilon}=-\vec{E} \cdot \operatorname{grad}(\log \varepsilon) .
$$

Using $\varepsilon=n^{2}$, simplification of Maxwell's equations gives

$$
\nabla^{2} \vec{E}+k^{2} n^{2} \vec{E}+2 \operatorname{grad}(\vec{E} \cdot \operatorname{grad} \log n)=0,
$$

where $n$ is refractive index, $k=\omega / c$ is the wave number associated with the frequency $\omega$, and $c$ is the speed of light in vacuum. The refractive index is expressed as sum of mean, and a fluctuating part that is the instantaneous refractive index is given by

$$
n=\bar{n}+n_{1}
$$

where $n_{1}$ is the deviation of $n$ from its mean value $\bar{n}$.

Therefore,

$$
\begin{aligned}
\nabla^{2} \vec{E}+k^{2}\left(\bar{n}+n_{1}\right)^{2} \vec{E} & +2 \operatorname{grad}\left(\vec{E} \cdot \operatorname{grad} \log \left(\bar{n}+n_{1}\right)\right)=0, \\
\nabla^{2} \vec{E}+k^{2} \bar{n}^{2} \vec{E}= & -2 \operatorname{grad}\left(\vec{E} \cdot \operatorname{grad} \log \left(\bar{n}+n_{1}\right)\right) \\
& -k^{2}\left(n_{1}^{2}+2 n_{1} \bar{n}\right) \vec{E} .
\end{aligned}
$$

As $\left|n_{1}\right| \ll 1$, it can be approximated that $\left(n_{1}^{2}+2 n_{1} \bar{n}\right) \approx 2 n_{1} \bar{n}$

$$
\begin{gathered}
\operatorname{grad}\left(\log \left(\bar{n}+n_{1}\right)\right)=\operatorname{grad}(\log \bar{n})+\operatorname{grad}\left(\log \left(1+\frac{n_{1}}{\bar{n}}\right)\right) \\
\operatorname{grad}(\log \bar{n})+\operatorname{grad}\left(\log \left(1+\frac{n_{1}}{\bar{n}}\right)\right) \\
\approx 0+\operatorname{grad}\left(\frac{n_{1}}{\bar{n}}-\frac{1}{2}\left(\frac{n_{1}}{\bar{n}}\right)^{2}\right) .
\end{gathered}
$$

After neglecting the square term, we get

$$
\begin{gathered}
\operatorname{grad}(\log \bar{n})+\operatorname{grad}\left(\log \left(1+\frac{n_{1}}{\bar{n}}\right)\right) \approx \frac{1}{\bar{n}} \operatorname{grad}\left(n_{1}\right), \\
\begin{aligned}
\nabla^{2} \vec{E}+k^{2} \bar{n}^{2} \vec{E}= & -\frac{2}{\bar{n}} \operatorname{grad}\left(\vec{E} \cdot \operatorname{grad} n_{1}\right) \\
& -2 \bar{n} n_{1} k^{2} \vec{E} .
\end{aligned}
\end{gathered}
$$

Equation (7) can be written in simplified form as

$$
\nabla^{2} u+k^{2} u=f(\vec{r})
$$

where $u$ can denote any of the field components and $f(\vec{r})$ the scattering potential, which is given by the expression

$$
f(r)=-\frac{2}{\bar{n}} \operatorname{grad}\left(\vec{E} \cdot \operatorname{grad} n_{1}\right)-2 \bar{n} n_{1} k^{2} \vec{E}
$$

It is well known that the solution of (8) corresponding to outgoing is given by

$$
u(r)=-\frac{1}{4 \pi} \int_{V} \frac{f\left(r^{\prime}\right) e^{i k\left(r-r^{\prime}\right)}}{\left|r-r^{\prime}\right|} d V^{\prime}
$$

where $\vec{r}^{\prime}$ is a variable vector ranging over the scattering volume $V$. It is shown in the appendix that under approximation (10) can be transformed to

$$
u(r) \approx \frac{1}{4 \pi} \frac{e^{i k r}}{r} \int_{V} f\left(\vec{r}^{\prime}\right) e^{-i k \vec{m} \cdot \vec{r}^{\prime}} d V^{\prime} .
$$


Therefore, from (9) and (11), we get

$$
\begin{aligned}
u(r) \approx & \frac{2 k^{2}}{4 \pi} \frac{e^{i k r}}{r} \\
& \times \int_{V}\left(\bar{n} n_{1} \vec{E}+\frac{1}{\bar{n}} \operatorname{grad}\left(\vec{E} \cdot \operatorname{grad} n_{1}\right)\right) \\
& \times e^{-i k \vec{m} \cdot \vec{r}^{\prime}} d V^{\prime}, \\
u(r) \approx & \frac{\bar{n} k^{2}}{2 \pi} \frac{e^{i k r}}{r} \int_{V} n_{1} \vec{E} e^{-i k \vec{m} \cdot \vec{r}^{\prime}} d V^{\prime} \\
& +\frac{k^{2}}{2 \pi \bar{n}} \frac{e^{i k r}}{r} \times \int_{V} \operatorname{grad}\left(\vec{E} \cdot \operatorname{grad} n_{1}\right) e^{-i k \vec{m} \cdot \vec{r}^{\prime}} d V^{\prime} .
\end{aligned}
$$

Using Gauss' theorem, under vanishing surface integral,

$$
\begin{aligned}
& \operatorname{grad}\left(\vec{E} \cdot \operatorname{grad} n_{1}\right) e^{-i k \vec{m} \cdot \vec{r}^{\prime}} \\
& =\left(\vec{E} \cdot \operatorname{grad} n_{1}\right) \operatorname{grad} e^{-i k \vec{m} \cdot \vec{r}^{\prime}} \\
& =(-i k \vec{m}) e^{-i k \vec{m} \cdot \vec{r}^{\prime}} .
\end{aligned}
$$

Therefore,

$$
\begin{aligned}
u(r) \approx & \frac{\bar{n} k^{2}}{2 \pi} \frac{e^{i k r}}{r} \times \int_{V} n_{1}\left(\vec{r}^{\prime}\right) \vec{E} e^{-i k \vec{m} \cdot \vec{r}^{\prime}} d V^{\prime} \\
& +\frac{i k \vec{m} k^{2}}{2 \pi \bar{n}} \frac{e^{i k r}}{r} \times \int_{V}\left(\vec{E} \cdot \operatorname{grad} n_{1}\right) e^{-i k \vec{m} \cdot \vec{r}^{\prime}} d V^{\prime} \\
= & \frac{\bar{n} k^{2} e^{i k r}}{2 \pi r} C_{1}+\frac{i k^{3} \vec{m} e^{i k r}}{2 \pi r \bar{n}} C_{2},
\end{aligned}
$$

where

$$
\begin{gathered}
C_{1}=\int_{V} n_{1}\left(\vec{r}^{\prime}\right) \vec{E} e^{-i k \vec{m} \cdot \vec{r}^{\prime}} d V^{\prime}, \\
C_{2}=\int_{V} \vec{E} \cdot \operatorname{grad} n_{1}\left(\vec{r}^{\prime}\right) e^{-i k \vec{m} \cdot \vec{r}^{\prime}} d V^{\prime} .
\end{gathered}
$$

Both terms of (14) represent spherical waves whose amplitudes and phases depend on the refractive index fluctuations inside the volume $V$ (through the random variables $C_{1}$ and $\mathrm{C}_{2}$ ). Indeed, second term can simply be ignored in calculating the flow of scattered energy. Consider

$$
u(r) \cong \frac{\bar{n} k^{2} e^{i k r}}{2 \pi r} C_{1} .
$$

The (spectral) intensity of the scattered field at any point $\vec{r}$, at frequency $\omega$, is just the diagonal element of the cross-spectral density of the scattered field and hence is given by

$$
\begin{aligned}
I(r, \omega)=\left(\frac{\bar{n} k^{2}}{2 \pi r}\right)^{2} \iint & \left(n_{1}\left(\vec{r}_{1}^{\prime}\right) n_{1}^{*}\left(\vec{r}_{2}^{\prime}\right)\right) \\
& \times\left(\vec{E}_{1} \vec{E}_{2}^{*}\right) e^{-i k \vec{m} \cdot\left(\vec{r}_{1}-\vec{r}_{2}\right)} d V_{1} d V_{2}^{*} .
\end{aligned}
$$

If the refractive index $n(\vec{r}, \omega)$ of the medium is a random function of position, then scattering potential $F(\vec{r}, \omega)$ will also be a random function of position, and the corresponding expressions for the cross-spectral density and for the spectral intensity of the scattered field are obtained at once by averaging over the ensemble of the scattering potential (denoted by angular brackets with subscript $n$ ).

Let $C_{n}\left(\vec{r}_{1}, \vec{r}_{2}\right)=C_{n}\left(\vec{r}_{1}-\vec{r}_{2}\right)$ denote the two-point spatial correlation function of the refractive index fluctuations, namely,

$$
C_{n}\left(r_{1}, r_{2}, \omega\right)=\left\langle n_{1}\left(\vec{r}_{1}\right) n_{1}^{*}\left(\vec{r}_{2}\right)\right\rangle,
$$

where the angle brackets represent the statistical average, taken over the ensemble $n_{1}(r, \omega)$ of the refractive index. Consider

$$
C_{n}\left(\vec{r}_{1}, \vec{r}_{2}\right)=C_{n}\left(\vec{r}_{1}-\vec{r}_{2}\right)
$$

Thus, by introducing the change of variables $\vec{r}_{1}-\vec{r}_{2}=\vec{\rho}$ and $\vec{r}_{1}+\vec{r}_{2}=2 \vec{r}$, we have

$$
I(r, \omega)=\left\{\frac{\bar{n} k^{2}}{2 \pi r}\right\}^{2} \int C_{n}(\vec{\rho})|\vec{E}|^{2} e^{-i k \vec{m} \cdot \vec{\rho}} d V_{\rho} .
$$

This formula shows explicitly how the spectral intensity of the scattered radiation depends on the correlation function of the refractive index fluctuations and on the field passing through it.

$$
\begin{aligned}
& \text { For } \vec{E}=\vec{A}_{0} \exp (i \vec{k} \cdot \vec{r}) \\
& \qquad I(r, \omega)=\left\{\frac{\bar{n} A_{0} k^{2}}{2 \pi r}\right\}^{2} \int C_{n}(\vec{\rho}) e^{i[\vec{k}-k \vec{m}] \cdot \vec{\rho}} d V_{\rho} .
\end{aligned}
$$

The spectral intensity is in fact Fourier transform of correlation function of the refractive index fluctuations. Thus, information about correlation function of the refractive index is important for the spectral intensity variant. This analysis applies only to a single frequency component of the field however, it can be extended to multiple or broad bandwidth.

\section{Results and Discussions}

A spectral intensity fluctuation from a liquid gain medium of a pulsed dye laser is known for a long time. Fluorescent dye dissolved in solvent is optically excited by high-power pulsed optical sources such as flash lamp or laser. The pump beam is absorbed in the dye medium following the exponential absorption law, and, typically, about $25 \%$ of the absorbed pump light energy is converted into heat [21]. The generated heat induces nonuniformity in the temperature, which gives rise to temperature gradients [22] and hence refractive index gradients in the solution that cause optical inhomogeneities in the medium. The temperature gradient caused by nonuniform heating of the dye solution was studied [22]. This inhomogeneity increases with pulse repetition frequency and the energy of the excitation source. The fluctuation of pump beam intensity induces differential heating of dye solution during different pulses. The nonuniform changes in 
the refractive index gradient of the dye solution lead to the refractive index fluctuations.

Sharma et al. [23, 24] have reported the experimental observation of intensity variation of fluorescence of Rhodamine $6 \mathrm{G}$ dye in liquid solvent excited by copper vapor laser, operating at $5.6 \mathrm{kHz}$ pulse repetition frequency in stationary medium. They recorded a number of spectra to see the thermal effects on the intensity variation of the fluorescence emission in the stationary dye solution. Figure 1 shows the spectral intensity variation [23] of the dye fluorescence in the field of thermal inhomogeneity. The spectral intensity decreases with increasing thermal inhomogeneity. The thermal inhomogeneities increase with time due to heat generated by the consecutive pulse of the excitation source. Consequently, the spatial correlation function of the refractive index varies, which in turn changes the spectral intensity of the emission passing through it. In stationary dye solution, an instantaneous refractive index in thermal field is given by

$$
n=\bar{n}+\frac{d n}{d T} \Delta T
$$

where $d n / d T$ is the refractive index gradient of the solvent, $\Delta T$ is the change in temperature. The fluctuating part $n_{1}$ of the refractive index is caused predominantly by microstructure in the spatial fluctuations of temperature. Thus, the fluctuating part of the refractive index in thermally dominated medium is given by

$$
n_{1}=\frac{d n}{d T} \Delta T
$$

The temperature rise per pulse can be approximated as

$$
\Delta T=\frac{a \alpha P}{\rho c_{p} l h d},
$$

where $a$ is a constant whose value depends on the nature of spatial/temporal pulse profile (i.e., Gaussian, Lorentzian, etc.), $m$ is mass of the dye solution, $\rho$ is the density, $c_{p}$ is specific heat of the dye solution, $l$ and $h$ are the length and the height of spot illuminated by the pump beam, $d$ is the penetration depth of the pump light inside the dye medium, $P$ is the optical excitation power, and $\alpha$ is the fraction of the excitation energy which is converted into heat. Therefore, spectral intensity variations of the observed fluorescence emission in the thermal field enlighten the variation in correlation function of the refractive index fluctuations of the medium.

To play down the thermal inhomogeneities of the medium, the dye volume exposed by the pump laser is generally rapidly replaced between successive pump laser pulses [25, 26]. For this purpose, the dye solution must be flown at sufficiently large velocities across the pump region. This often results in chaos/turbulence and hence degradation of the optical quality of the medium $[21,22,27]$. The propagation of the spontaneously emitted fluorescent photons is affected by the degraded qualities of the medium. The estimated Reynolds numbers [24] were 90.6, 181.2, ..., and 1630.8 for

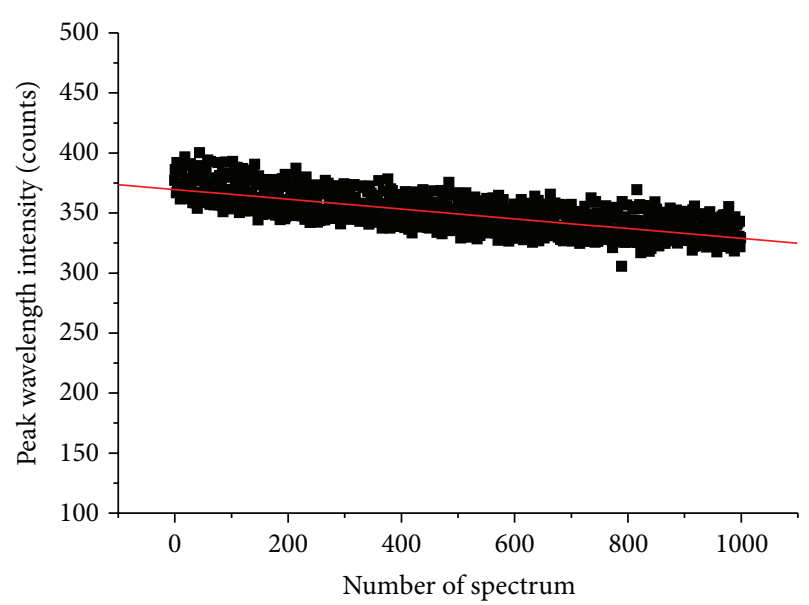

FIGURE 1: The spectral intensity variation [23] of fluorescence in the field of thermal inhomogeneity.

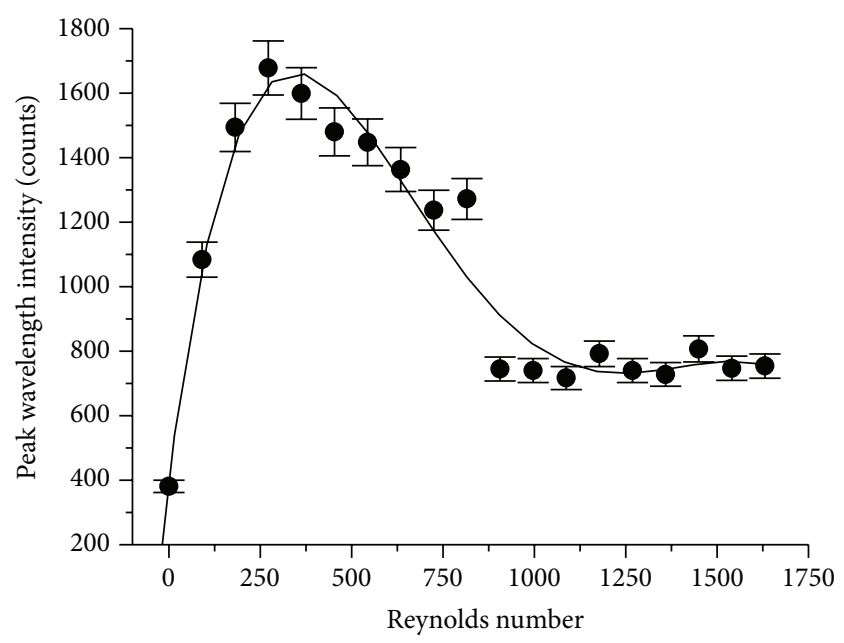

FIGURE 2: The spectral intensity variation of the emission data [24] and fitted function with the Reynolds numbers of the liquid flow.

the flow rates of $1,2, \ldots$, and 18 liter per minute (lpm), respectively. The spectral intensity of the fluorescent photons emitted in the chaotically sullied medium, observed outside the medium, at flow rates from 1 to $18 \mathrm{lpm}$, shows variation [24]. Figure 2 shows the spectral intensity variation of the emission data [24] and fitted function with the Reynolds numbers of the liquid flow. The spectral intensity increases with increasing the flow rates. It is probably due to attenuation in the spatial correlation function of the induced thermal refractive index fluctuations. Figure 3 shows the spectral intensity variation of the emission data [24] with fitted polynomial at low Reynolds numbers. Further increase in the flow rates decreases the intensity. Figure 4 shows the spectral intensity variation of the Gaussian fitted data at higher Reynolds numbers. It is perhaps because of the spatial correlation function of the flow-induced eddies/turbulences and hence spatial refractive index fluctuations in the medium. The spatial correlation function of the refractive index of 


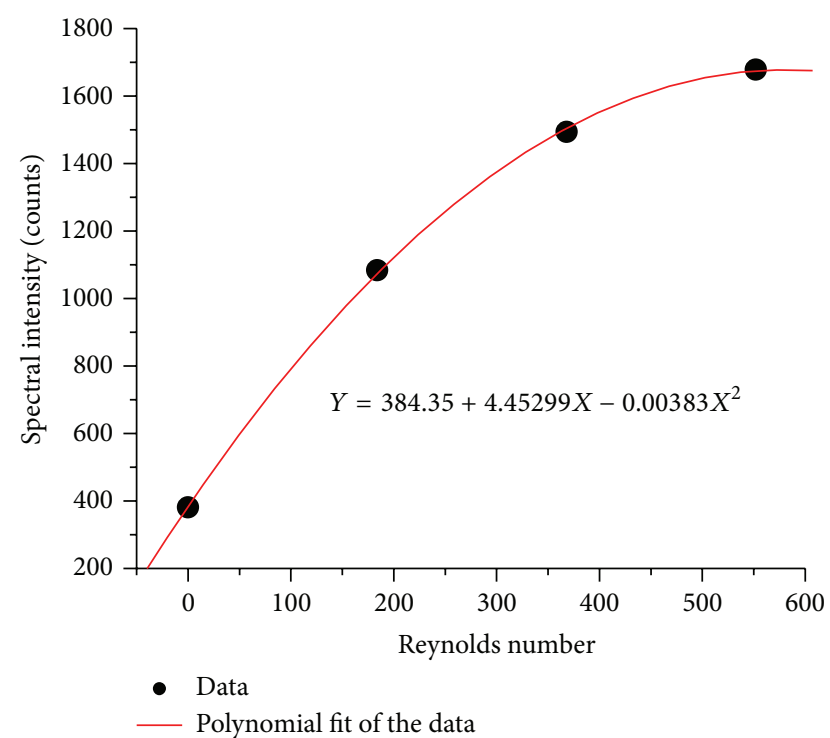

FIGURE 3: The spectral intensity variation of the emission data [24] with fitted polynomial at low Reynolds numbers.

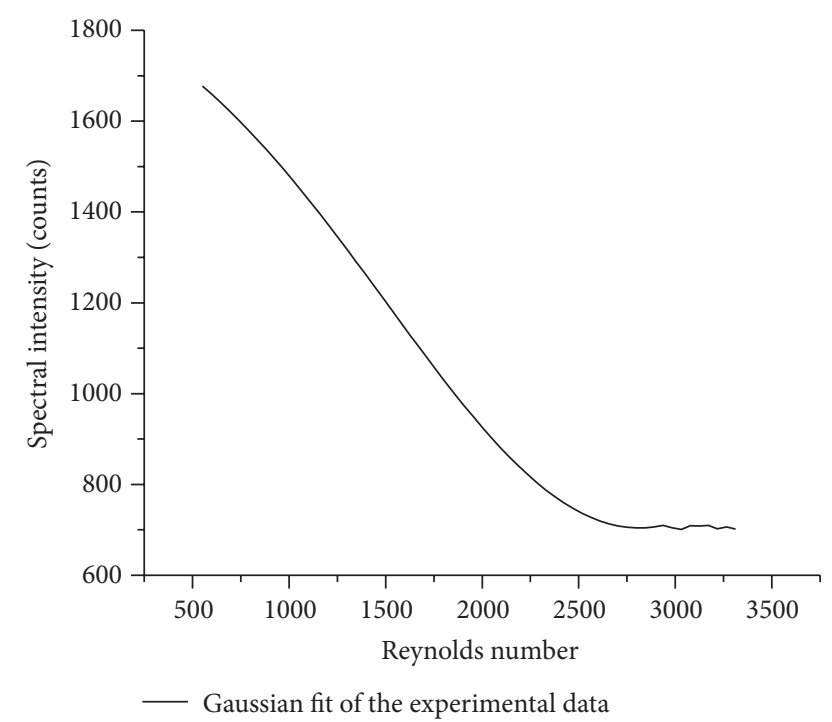

Figure 4: The spectral intensity variation of the fitted Gaussian at higher Reynolds numbers.

the liquid medium, at higher Reynolds numbers, can be approximated in the form

$$
C_{n}(\vec{\rho})=C_{n}(0) \exp \left[-\beta\left(\frac{\rho}{\rho_{0}}\right)^{2}\right],
$$

where $\beta$ and $\rho_{0}$ are positive constants, which depends upon the scale length and nature of the inhomogeneity.

In another similar study [28] related to fluorescence fluctuation, it was observed that the spectral intensity along with its fluctuations varies in static and dye solution flow medium. It varies from 10,000 to 70,000 counts in static solution. The value is large, above 80,000 counts in flowing medium. Figure 5 shows the spectral intensity variation of fluorescence

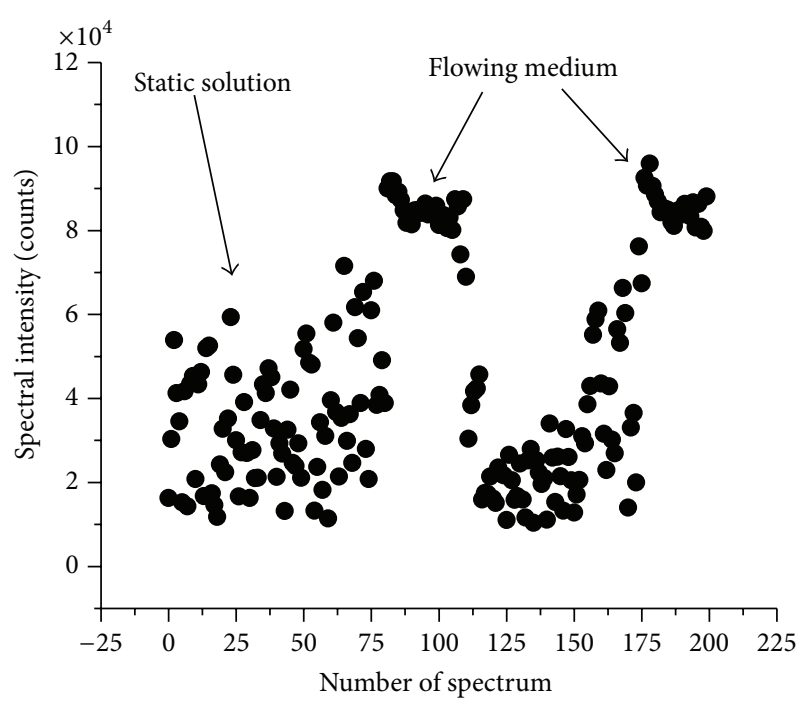

FIGURE 5: The spectral intensity variation of fluorescence emission in static and flowing dye medium.

emission in static and flowing dye medium. Large fluctuations, induced by the thermal field, in fluorescence intensity in static dye solution are due to the random variation in the scale length of correlation function of the refractive index fluctuations. Minimization of the thermal inhomogeneity, by flow of the medium, increases the spectral intensity. Varnado [27] has observed degradation of flash lamp pumped dye laser output energy with increasing flow velocity and reported that the degradation is caused by scattering centers present in the dye after flowing. The decreased intensity was, in fact, due to refractive index variation. Variation of backscattered intensity with optical path length from a random medium [29], described by optical path length spectroscopy, can also be appreciated by refractive index correlation function variation.

\section{Conclusions}

In conclusion, it was established that macroscopic theory of propagation and scattering of light through random media can be functional for the dye liquid flowing media in microscopic level, with the modest approximations. Spatial correlation function known for a long time has been correlated with refractive index fluctuations of the flowing dye medium. Analytical expression for the spectral intensity of the scattered radiation and correlation function of the refractive index of the medium was formulated. It was shown that the spectral intensity of the field scattered by random medium depends on the correlation function of the refractive index fluctuations of the flowing dye liquid medium. Experimentally observed spectral intensity variation of the fluorescence emission of dye and the dye laser emission in liquid media in the presence of thermal and flow field is a corollary of scale length of spatial refractive index unsteadiness. Spectral intensity scattering of the laser emission in liquid flowing media is directly 
coupled with spatial correlation function of the refractive index fluctuations.

\section{Appendix}

\section{Derivation of an Approximation Relating to Expression (10)}

We derive here the approximation that is applied in expression (10), namely, we choose the origin of coordinates inside the scattering volume. If the observation point $\vec{r}$ is at a great distance from the scattering volume $V$ as compared to the dimensions of $V$, then for all $\vec{r}^{\prime}$ the quantity $\left|\vec{r}-\vec{r}^{\prime}\right|$ is almost constant and close to $r=|\vec{r}|$. In this case, the quantity $\left|\vec{r}-\vec{r}^{\prime}\right|$ can be expanded in a series of powers that is

$$
\left|\vec{r}-\vec{r}^{\prime}\right|=r-\vec{m} \cdot \vec{r}^{\prime}+\frac{1}{2 r}\left[r^{\prime 2}-\left(\vec{m} \cdot \vec{r}^{\prime}\right)^{2}\right]+\cdots,
$$

where $\vec{m}=\vec{r} / r$ is a unit vector directed from the origin of coordinates (chosen within the scattering volume) to the observation point. If the inequality

$$
\frac{k}{2 r}\left[r^{\prime 2}-\left(\vec{m} \cdot \vec{r}^{\prime}\right)^{2}\right] \ll 1
$$

holds for all values of $r^{\prime}$, that is, if the dimensions $L$ of the scattering volume satisfy the condition $\lambda r \gg L^{2}$, then

$$
\exp \left(i k\left|\vec{r}-\vec{r}^{\prime}\right|\right) \approx \exp \left[i k\left(r-\vec{m} \cdot \vec{r}^{\prime}\right)\right] .
$$

Moreover, in the denominator $\left|\vec{r}-\vec{r}^{\prime}\right|$ can be replaced by $r$. Thus, we have in far field zone

$$
u(r) \approx \frac{1}{4 \pi} \frac{e^{i k r}}{r} \int_{V} f\left(\vec{r}^{\prime}\right) e^{-i k \vec{m} \cdot \vec{r}^{\prime}} d V^{\prime},
$$

which is expression (11).

\section{Acknowledgments}

The author wishes to acknowledge M. P. Singh for the discussion and significant suggestions. The author is also thankful to H. S. Vora and S. B. Roy for their guidance and encouragement to the work.

\section{References}

[1] L. L. Foldy, "The multiple scattering of waves. I. General theory of isotropic scattering by randomly distributed scatterers," Physical Review, vol. 67, no. 3-4, pp. 107-119, 1945.

[2] M. Lax, "Multiple scattering of waves," Reviews of Modern Physics, vol. 23, no. 4, pp. 287-310, 1951.

[3] L. A. Chernov, Wave Propagation in a Random Medium, McGraw-Hill, New York, NY, USA, 1960.

[4] V. I. Tatarskii, Wave Propagation in a Turbulent Medium, McGraw-Hill, New York, NY, USA, 1961.

[5] J. B. Keller, "Stochastic equations and wave propagation in random media," in Proceedings of Symposia in Applied Mathematics, vol. 13, pp. 145-147, American Mathematical Society, Providence, RI, USA, 1964.
[6] M. J. Beran, "Propagation of the mutual coherence function through random media," The Journal of the Optical Society of America, vol. 56, no. 11, pp. 1475-1480, 1966.

[7] V. I. Tatarskii and M. E. Gertsenshtein, "Propagation of waves in a medium with strong fluctuation of the refractive index," Journal of Experimental and Theoretical Physics, vol. 17, pp. 548$563,1967$.

[8] Z. I. Feizulin and Y. A. Kravtsov, "Broadening of a laser beam in a turbulent medium," Radiophysics and Quantum Electronics, vol. 10, no. 1, pp. 33-35, 1967.

[9] P. Bassanini, "Wave propagation in a one-dimensional random medium," Radio Science, vol. 2, pp. 429-436, 1967.

[10] A. Ishimaru, "Fluctuations of a beam wave propagating through a locally homogeneous medium," Radio Science, vol. 4, no. 4, pp. 295-305, 1969.

[11] A. Ishimaru, Wave Propagation and Scattering in Random Media, vol. 1-2, Academic Press, San Diego, Calif, USA, 1978.

[12] R. L. Fante, "Wave propagation in random media: a systems approach," in Progress in Optics, E. Wolf, Ed., vol. 22, pp. 341398, Elsevier, Amsterdam, The Netherlands, 1985.

[13] J. Wu, "Propagation of a Gaussian-Schell beam through turbulent media," Journal of Modern Optics, vol. 37, no. 4, pp. 671-684, 1990.

[14] J. Wu and A. D. Boardman, "Coherence length of a Gaussian Schell-model beam and atmospheric turbulence," Journal of Modern Optics, vol. 38, pp. 1355-1363, 1991.

[15] A. Ishimaru, Electromagnetic Wave Propagation, Radiation, and Scattering, Prentice-Hall, Englewood Cliffs, NJ, USA, 1991.

[16] L. C. Andrews and R. L. Phillips, Laser Beam Propagation through Random Media, SPIE Press, Bellingham, Wash, USA, 1998.

[17] M. Santarsiero, F. Gori, R. Borghi, G. Cincotti, and P. Vahimaa, "Spreading properties of beams radiated by partially coherent Schell-model sources," Journal of the Optical Society of America A, vol. 16, no. 1, pp. 106-112, 1999.

[18] J. Jannson, T. Jannson, and E. Wolf, "Spatial coherence discrimination in scattering," Optics Letters, vol. 13, no. 12, pp. 1060-1062, 1988.

[19] T. D. Visser, D. G. Fischer, and E. Wolf, "Scattering of light from quasi-homogeneous sources by quasi-homogeneous media," Journal of the Optical Society of America A, vol. 23, no. 7, pp. 1631-1638, 2006.

[20] G. Gbur and E. Wolf, "Spreading of partially coherent beams in random media," Journal of the Optical Society of America A, vol. 19, no. 8, pp. 1592-1598, 2002.

[21] C. S. Zhou, "Design of a pulsed single-mode dye laser," Applied Optics, vol. 23, no. 17, pp. 2879-2885, 1984.

[22] M. Amit, G. Bialolenker, D. Levron, and Z. Burshtein, "Refractive index gradients and dye solution flow characteristics in pulsed copper vapor laser pumped dye laser cells," Journal of Applied Physics, vol. 63, no. 5, pp. 1293-1298, 1988.

[23] N. Sharma, N. Singh, H. S. Vora, and S. L. Goyal, "Influence of the medium on the fluorescence of copper vapor laser pumped rhodamine $6 \mathrm{G}$ dye: introduction, experimental details and stationary case," Optics Journal, vol. 1, pp. 13-17, 2007.

[24] N. Sharma, N. Singh, H. S. Vora, and S. L. Goyal, "Influence of the medium on the fluorescence of copper vapor laser pumped rhodamine 6G dye: addendum," Optics Journal, vol. 1, pp. 18-22, 2007.

[25] F. J. Duarte and L. W. Hillman, Dye Laser Principle, Academic Press, New York, NY, USA, 1990. 
[26] C. E. Webb, "High-power dye lasers pumped by copper vapor lasers," in High-Power Dye Lasers, F. J. Duarte, Ed., pp. 143-182, Springer, Berlin, Germany, 1991.

[27] S. G. Varnado, "Degradation in long-pulse dye laser emission under fast-flow conditions," Journal of Applied Physics, vol. 44, no. 11, pp. 5067-5068, 1973.

[28] N. Singh, H. K. Patel, S. K. Dixit, and H. S. Vora, "Fluorescence fluctuation of Rhodamine $6 \mathrm{G}$ dye for high repetition rate laser excitation," Journal of Luminescence, vol. 134, pp. 607-613, 2013.

[29] G. Popescu and A. Dogariu, "Optical path-length spectroscopy of wave propagation in random media," Optics Letters, vol. 24, no. 7, pp. 442-444, 1999. 

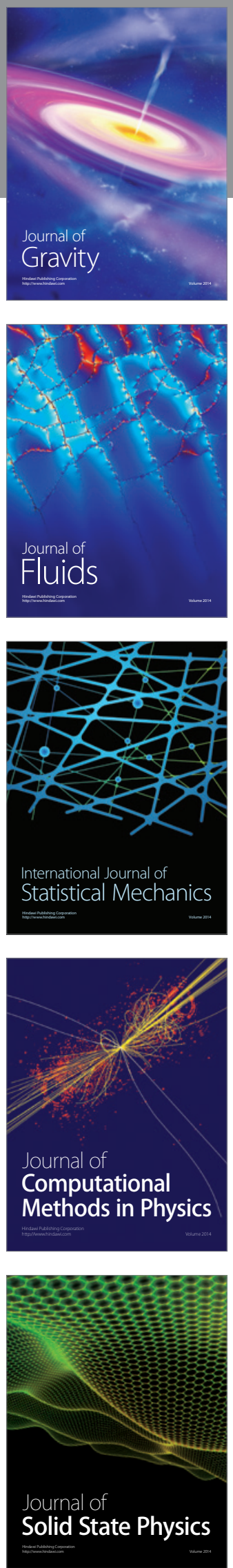

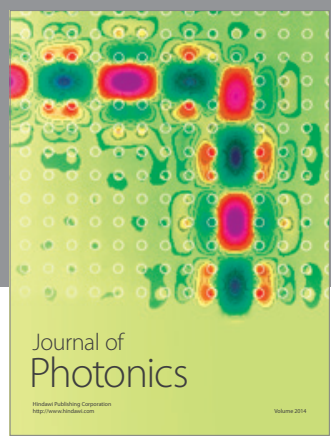

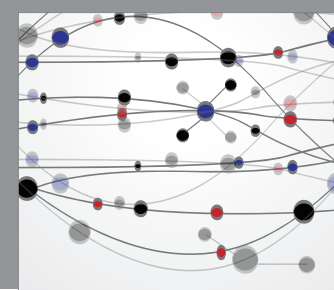

The Scientific World Journal

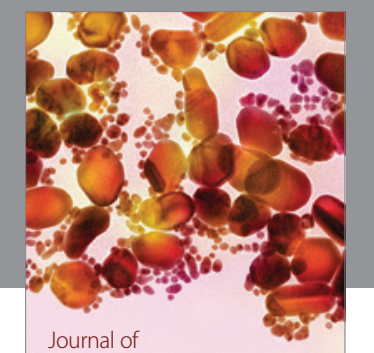

Soft Matter
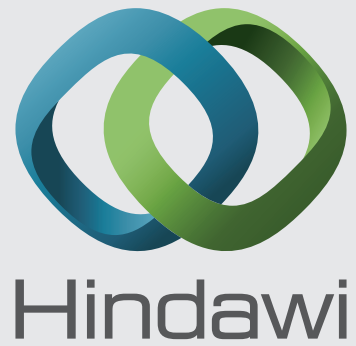

Submit your manuscripts at

http://www.hindawi.com
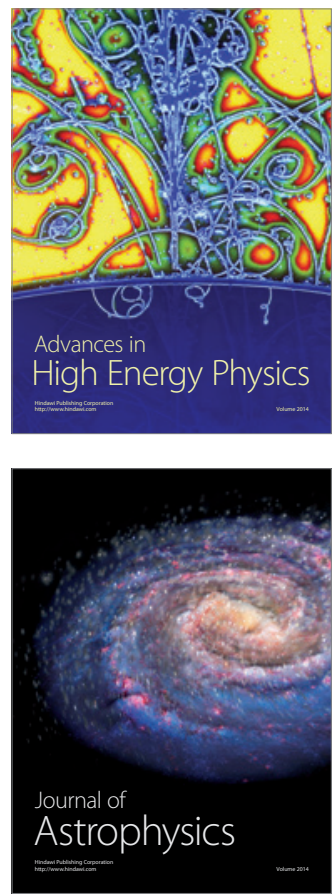
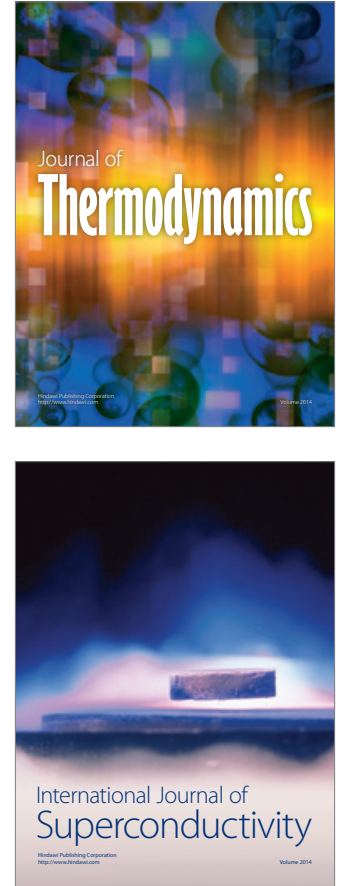
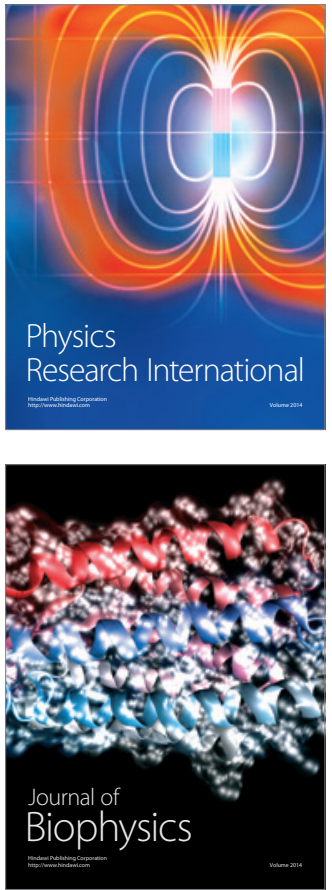
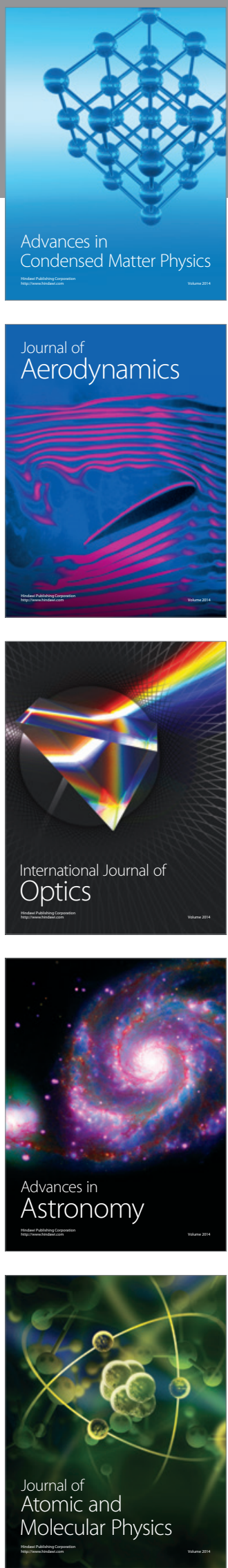